\title{
Image-plane fringe tracker for adaptive-optics assisted long baseline interferometry
}

Michael J. Ireland, Denis Defrère, Frantz Martinache, John D. Monnier, Barnaby Norris, et al.

Michael J. Ireland, Denis Defrère, Frantz Martinache, John D. Monnier, Barnaby Norris, Peter Tuthill, Julien Woillez, "Image-plane fringe tracker for adaptive-optics assisted long baseline interferometry," Proc. SPIE 10701, Optical and Infrared Interferometry and Imaging VI, 1070111 (9 July 2018); doi: $10.1117 / 12.2314393$

Event: SPIE Astronomical Telescopes + Instrumentation, 2018, Austin, Texas, United States 


\title{
Image-plane fringe tracker for adaptive-optics assisted long baseline interferometry
}

\author{
Michael J. Ireland ${ }^{\mathrm{a}}$, Denis Defrère ${ }^{\mathrm{b}}$, Frantz Martinache ${ }^{\mathrm{c}}$, John D. Monnier ${ }^{\mathrm{d}}$, Barnaby Norris ${ }^{\mathrm{e}, \mathrm{f}}$, \\ Peter Tuthille, and Julien Woillez ${ }^{\mathrm{g}}$ \\ ${ }^{a}$ Research School of Astronomy and Astrophysics, Australian National University, Canberra, \\ ACT 2611, Australia \\ ${ }^{\mathrm{b}}$ Space sciences, Technologies \& Astrophysics Research (STAR) Institute, Université de Liège, \\ 19c allée du Six Aout, bât B5c, B-4000 Liège, Belgium \\ 'Université Côte d'Azur, Observatoire de la Côte d'Azur, CNRS, Laboratoire Lagrange, France \\ ${ }^{\mathrm{d}}$ Department of Astronomy, University of Michigan, Ann Arbor, MI, 48109 USA \\ eSydney Institute for Astronomy and Sydney Astrophotonic Instrumentation Laboratories, \\ School of Physics, University of Sydney, NSW 2006, Australia \\ ${ }_{\mathrm{f}}$ Australian Astronomical Observatory, Faculty of Science and Engineering, Macquarie \\ University, NSW 2109, Australia \\ ${ }^{g}$ European Southern Observatory, Karl-Schwarzschild-Str. 2, 85748 Garching bei München, \\ Germany
}

\begin{abstract}
Accurate fringe tracking is essential for sensitive long-wavelength thermal background limited operation of the current Very Large Telescope Interferometry (VLTI) and future Planet Formation Imager (PFI) facilities. We present and simulate a dual fringe tracking and low-order adaptive optics concept based on a combination of non-redundant aperture interferometry and eigenphase in asymmetric pupil wavefront sensing. This scheme can acquire fringes at many wavelengths of path length offset between telescopes, even with moderate tilt offset and pupil shifts between beams. Once locked to fringes, our technique can also be used for simultaneous low-order wavefront sensing, and has near-optimum sensitivity where there is a dominant point-source image component. This concept is part of the Heimdallr visitor instrument currently being investigated for VLTI.
\end{abstract}

Keywords: Long baseline optical interferometry, fringe tracking, adaptive optics

\section{INTRODUCTION}

Modern long baseline optical interferometers include adaptive optics systems at each telescope, a lossy beam train (with near infrared efficiencies in the range 0.2 to 0.5 ) followed by some combination of fringe acquisition and tracking system and scientific beam combiner. The fringe tracker can either have high residuals, finding the group delay position within the coherence envelope $\lambda^{2} / \Delta \lambda$ of the science beam combiner or residuals of order 1 radian of fringe phase. Reducing fringe tracking residuals below a radian of fringe phase is often seen as a way to achieve long integrations in the presence of detector noise, ${ }^{1}$ but measuring fringe phase with a sub-radian accuracy has a much more fundamental application in enabling coherent integration ${ }^{2}$ of differential quantities such as closure-phase, wavelength-differential phase ${ }^{3}$ and dual fringe packet astrometry. ${ }^{4,5}$

Several future interferometer concepts, such as high-contrast imaging in the mid-infrared with VLTI, ${ }^{6}$ or the $\mathrm{PFI}^{7}$ require high accuracy fringe tracking in order to operate. In fact, recent work on the concept of kernel nulling ${ }^{8}$ has shown that nulling interferometry contrasts are often proportional to the cube of the fringe tracking residual, and require residuals smaller than $50 \mathrm{~nm}$ in order to achieve key high-contrast exoplanet detection goals.

M.J.I: michael.ireland@anu.edu.au

Optical and Infrared Interferometry and Imaging VI, edited by Michelle J. Creech-Eakman,

Peter G. Tuthill, Antoine Mérand, Proc. of SPIE Vol. 10701, 1070111 · c) 2018 SPIE

CCC code: $0277-786 \mathrm{X} / 18 / \$ 18 \cdot$ doi: $10.1117 / 12.2314393$

Proc. of SPIE Vol. 10701 1070111-1 
Table 1. Idealised adaptive optics sensitivity for $\mathrm{H}+\mathrm{K}$ Fringe Tracking at $1.5 \mu \mathrm{m}$ Strehl $=0.6$

\begin{tabular}{lllll}
\hline Configuration Name & $\begin{array}{l}\text { Telescope Diameter } \\
(\mathrm{m})\end{array}$ & $\begin{array}{l}\text { Bandpass } \\
(\mu \mathrm{m})\end{array}$ & $\begin{array}{l}\text { Detected Flux } \\
\text { Photons } / \mathrm{s} / \mathrm{m}^{2}\end{array}$ & $\begin{array}{l}\text { Magnitude Limit } \\
(\text { Vega })\end{array}$ \\
\hline VLTI UTs & 8.0 & $0.6-0.9$ & $8 \times 10^{4}$ & 12.3 \\
VLTI ATs & 1.8 & $0.6-0.9$ & $7 \times 10^{4}$ & 12.5 \\
PFI & 3.0 & $0.7-1.3$ & $1.2 \times 10^{5}$ & 11.7
\end{tabular}

One of the key limitations to many fringe tracking concepts is the problem of flux dropouts in a spatially filtered system, in turn caused by wavefront aberrations. In the case of a system with tip/tilt correction, it is the higher order modes that cause drop-outs. However, an AO system with a sensor noise (rather than actuation) limit to correction is often limited by tip/tilt. ${ }^{9}$ In this paper, we propose that the adaptive optics system and fringe tracker should be seen as a more integrated system that provides a high spatial cohrerence beam to the science combiner, also enabling low spectral resolution science. Note that we are not considering piston reconstruction in this paper, ${ }^{10}$ which is an separate but also highly valuable reason to consider fringe tracking and adaptive optics as an integrated system.

In section 2 we describe some analytic considerations of fringe tracking sensitivity. In section 3 we describe a particular instrument concept (Heimdallr for VLTI), and in section 4 we describe the simulations and algorithms in more detail, with particular applicability to the Heimdallr concept. Finally, we discuss further work needed in section ??.

\section{ANALYTIC SENSITIVITY CONSIDERATIONS}

Prior to describing the details of the image plane fringe tracker, we will first outline some generic principles of adaptive optics and fringe-tracking sensitivity, with specific reference to the VLTI using the Auxilliary Telescopes (ATs) and the Unit Telescopes (UTs), and to the baseline Planet Formation Imager (PFI) concept using $3 \mathrm{~m}$ telescopes. We will show that, so long as an all-in-one fringe tracker is not limited by readout noise, the limiting sensitivity should generally be determined by the adaptive optics system (assuming $0.6-1 \mu \mathrm{m}$ operation) and not the fringe tracker operating at $1.5-2.4 \mu \mathrm{m}$ wavelengths.

For both adaptive optics and fringe tracking sensitivity calculations, we will use the analytic formalism of Ref. 9 to compute performance. In this formalism, the corrected amplitude as a function of spatial frequency (adaptive optics) or baseline (fringe tracking), telescope diameter, source brightness and parameters of atmospheric seeing is given as the quadrature sum of a phase lag and a shot-noise component. The system integration time $\Delta t$ is roughly optimal when these two terms are equal. We will consider the seeing corresponding to the mean seeing at Paranal, ${ }^{11}$ i.e. $0.86 "$, with $\tau_{0}=2.0 \mathrm{~ms}$ and a corresponding effective wind velocity $\bar{v}=18.4 \mathrm{~m} \mathrm{~s}^{-1}$. This value of $\tau_{0}$ has a $60-70 \%$ chance of being met on any given night. We will consider a nearly perfect detector with an excess noise factor $\alpha=1.25$ and detector readout noise of $0.2 \mathrm{e}-$, applicable to recent results on $72 \times 72$ sub-regions of a Saphira array. ${ }^{12}$

\subsection{Adaptive Optics Sensitivity}

Recent advances in adaptive optics has shown that stable Strehl ratios in extreme AO are possible, ${ }^{13}$ without drop-outs that have plagued many installed wavefronts sensors. In order to compute the required sensitivity for adaptive optics, we will assume a shot-noise sensitivity parameter ${ }^{9} \beta$ of 2.5 . This is roughly applicable to a low-modulation pyramid wavefront sensor, a Shack-Hartmann wavefront sensor operating at high spatial frequencies, or a curvature wavefront sensor operating at medium to high spatial frequencies. Note that all these typical wavefront sensors lose significant sensitivity to low order modes, especially tip/tilt. We also assume a throughput to the wavefront sensor of $40 \%$ (atmosphere plus 15 surfaces at $95 \%$ efficiency and a $90 \%$ quantum efficiency), and a deformable mirror correcting up to $3.3 \mathrm{cycles} / \mathrm{m}$, equivalent to a $0.15 \mathrm{~m}$ subaperture spacing. In order to achieve an RMS wavefront error of less than 0.7 radians at a shortest fringe tracking wavelength of $1.5, \mu \mathrm{m}$, with this formalism we compute the in-principle flux requirements as shown in Table 1.

These numbers are not far from typical expectations of large telescope adaptive optics systems. Note that operating an EMCCD in the mode of NAOMI with an excess noise factor of $\sqrt{2}$, equivalent to a $50 \%$ loss of quantum efficiency, loses an additional $\sim 0.75$ magnitudes compared to the numbers presented here. 


\subsection{Pairwise Fringe Tracking Sensitivity}

With the same formalism, we can compute a magnitude limit for pairwise beam combination by optimising a system integration time so that servo lag error equals fringe phase noise error. In this case, we will optimise so that the quadrature sum two is 0.5 radians, and assume a warm optics efficiency (including coupling/Strehl) of 0.15 and a cold efficiency of 0.22 , for a total efficiency of $3.3 \%$. This is a conservative assumption for a sensitivity-optimised fringe tracker, and a factor of $\sim 2$ lower than the modelled Heimdallr instrument efficiency or the PFI baseline design. We will use a background rate of 700 photons/s over a 1.5 to $2.4 \mu \mathrm{m}$ bandpass.

The fringe phase error in an ABCD scheme is given by:

$$
\sigma_{\phi, \mathrm{ABCD}}^{2}=\frac{2\left(\alpha(F \times A+B) \Delta t+N_{\mathrm{pix}} \sigma_{r o}^{2}\right)}{(F \times A)^{2} \Delta t^{2}},
$$

where $F$ is the stellar flux in photons $/ \mathrm{s} / \mathrm{m}^{2}, A$ the telescope area, $B$ the background flux in photons $/ \mathrm{s}, \sigma_{\text {ro }}$ the readout noise, $N_{\text {pix }}$ the number of pixels, and $\delta t$ the fringe integration time. The piston two-dimensional spatial power spectrum is:

$$
\Phi(\mathbf{k})=\frac{0.023}{r_{0}^{5 / 3}}\left(\frac{2 J_{1}(\pi D|\mathbf{k}|)}{\pi D|\mathbf{k}|}\right)^{2}|\mathbf{k}|^{-11 / 3}
$$

To obtain the frequency spectrum, we integrate this numerically along a direction perpendicular to the wind velocity:

$$
\Phi(f)=\frac{1}{\bar{v}} \int \Phi\left(\frac{f}{\bar{v}} \hat{\boldsymbol{e}}_{\|}+\kappa_{\perp} \hat{\boldsymbol{e}}_{\perp}\right) d \kappa_{\perp} .
$$

Finally, the time lag error in differential fringe phase in the long baseline limit is:

$$
\sigma_{\phi, \text { lag }}^{2}=16 \pi^{2} t^{2} \int_{0}^{\infty} f^{2} \Phi(f) d f
$$

For our canonical parameters, we obtain a half radian total phase error $\sqrt{\sigma_{\phi, \text { lag }}^{2}+\sigma_{\phi, \mathrm{ABCD}}^{2}}$ at an optimal integration time $t=10 \mathrm{~ms}$ and a corresponding flux of $F=3700$ detected photons per second. This corresponds to a mean Vega target correlated magnitude over the $\mathrm{H}$ and $\mathrm{K}$ bands for the ATs of 12.3, and substantially fainter for PFI or the UTs. An ideal fringe tracker operating on typical object with solar or redder colours $\left(m_{R}-m_{H}>0.8\right)$ should therefore be able to track fringes with low residuals on a marginally resolved source whenever an adaptive optics system can lock!

\subsection{Multi-Telescope Fringe Tracking Sensitivity}

In the last SPIE conference, the PFI status paper ${ }^{14}$ and the fringe tracking working group ${ }^{15}$ each compared signalto-noise of different beam combiner architectures. When imaging over a sufficiently wide field of view, an all-inone beam combiner architecture was preferred over pairwise combination, ${ }^{14}$ while when considering a detector readout-noise limited architecture, a hierarchical fringe tracker appeared preferred. ${ }^{15}$ In a shot-noise limited architecture, with high target visibilities and with signal-to-noise is high enough to consider combinations of baseline phases linearly, we can consider a much simpler version of fringe tracking sensitivity. When moving from a 2-telescope fringe tracker to an $\mathrm{N}_{T}$ telescope fringe tracker in an all-in-one configuration, we have $N_{T}\left(N_{T}-1\right) / 2$ total baselines, and therefore this many phase measurements. There are $N_{T}-1$ phase differences we have to solve for, so the fringe tracking phase noise is $\sqrt{2 / N_{T}}$ times the single baseline phase noise. The shot-noise per 
baseline is also increased by a factor of $\sqrt{N_{T} / 2}$, given that the background and stellar flux both increase by a factor of $N_{T} / 2$. This results in the overall fringe tracking phase sensitivity being independent of $N_{T}$.

This means that fringe tracking sensitivity in the shot-noise limited regime (i.e. negligible detector noise) no different for an all-in-one combiner, than either a single-baseline ABCD fringe tracker or the first stage of a hierarchical fringe tracker ${ }^{15}$ with $50 \%$ flux passed through (the highest fringe-tracking SNR configuration for an ideal hierarchical fringe tracker). The fringe-tracking concept described in the following section is shot-noise limited in almost all scenarios, so by this argument is not only highly versatile but a signal-to-noise optimal fringe tracker.

\section{HEIMDALLR INSTRUMENT FOR VLTI}

As a specific hardware example for this concept, we will describe the Heimdallr* instrument concept currently under review for funding by the Australian Research Council, and including an initial collaboration of institutes and Universities in Australia and Europe.

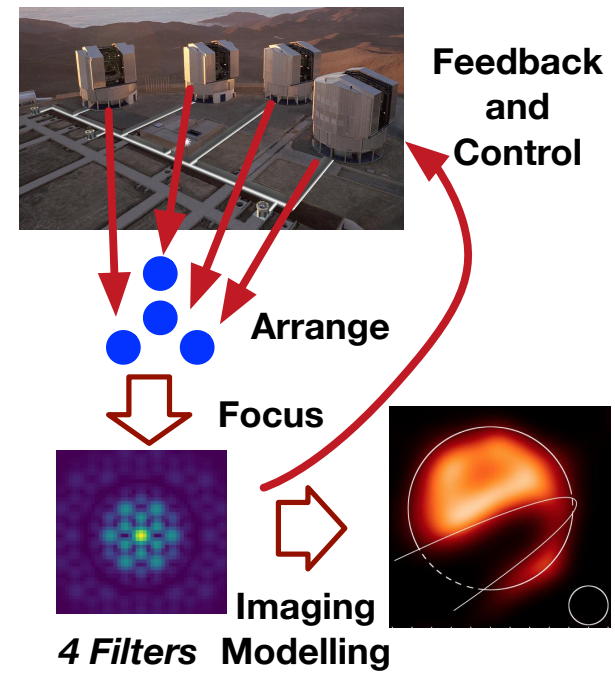

Figure 1. A conceptual diagram of Heimdallr, showing the arrangement (blue dots) of the 4 VLT beams which then form an image (interferogram) that contains information to both control the VLTI instrument and to yield calibrated interferometric quantities. The example image of a disk eclipsing a stellar surface comes from (CHARA): Heimdallr will enable imaging of Active Galactic Nuclei and inner disks of solar-type stars.

(1) wall both benefit firom this inovation, and extend it through the active detector control program underway at ANU.

As a multiaxial design, beams in Heimdallr are placed next to each other in a two-dimensional array with zero redundancy in separation, then brought to a common focus so that they are combined in the manner of an

${ }^{*}$ For those who like acronymns, Heimdallr could stand for the High-Efficiency Infrared Do-it-ALL Recombiner. As well as an acryonymn, Heimdallr is the guardian of the Bifröst bridge to Asgard, who can see over a hundred leagues and see night as it were day. Heimdallr is thus both a key character of Norse mythology and the first component of the long term vision of VLTI Kernel NulliNG (VIKiNG) within a broader European collaboration in the HI-5 framework. 
aperture-mask interferometer. This innovation delivers a number of advantages over previous VLTI instrumentation:

- Retention of the full two-dimensional information contained in the interferogram, providing high-sensitivity wavefront control. ${ }^{17}$

- Using this two-dimensional information to provide visibility calibration, rather than the lossy process of optical fiber injection.

- Multiple simultaneous wavelengths over a broad bandpass, avoiding the $2 \pi$ phase ambiguity in narrow wavelength fringe tracking. No previous VLTI instrument has used H+K filters simultaneously for fringetracking.

- Discrete filters (based on dichroic beamsplitters) instead of a spectrograph. This increases overall throughput compared to previously used designs.

- Using modern computers to their fullest by basing the real-time servo loop and analysis on 2D Fast Fourier transforms. Only $40 \mu$ s computation time with one dedicated core per wavelength represents a negligible servo lag.

- Broad bandwidths with by active dispersion compensation. This is enabled by thin moving ZnSe wedges as dispersion compensators. ${ }^{18}$

- Accurate closure-phase for simultaneous science using the proven technology of aperture mask interferometry.

Our design process has produced comprehensive simulations of the performance of Heimdallr, summarised in Table 2 as system requirements. Like the other newest 4-telescope beam combiners at VLTI, we will be able to reconstruct images given adequate sky coverage (i.e. observation time). Heimdallr will be designed to track on much lower visibility fringes than Gravity, enabling imaging of objects that take up the full field of view.

The optical layout of the warm and cold optics is shown in Figure 2, in which every component has a specification and either a vendor quote or a suitable off-the-shelf item. We have a complete optical design with vendor quotes, a cryogenic design which is a modified copy of a previous cryostat (Figure 3), and an end to end fringe tracking simulation including all noise sources including an imperfect AO system.

\section{FRINGE TRACKING ALGORITHMS}

We will consider several variants of fringe sensing algorithms, each which has their own advantages and disadvantages.

\subsection{Linear Assymetric Pupil Wavefront Sensor}

By directly using the assymetric pupil wavefront sensor, ${ }^{17}$ we can directly compute phase on a grid of discrete phases per sub-pupil Table 2. System Requirements for the Heimdallr VLTI instrument.

\begin{tabular}{l|l|l} 
Parameter & $\begin{array}{l}\text { Auxilliary } \\
\text { Telescopes }\end{array}$ & $\begin{array}{l}\text { Unit } \\
\text { Telescopes }\end{array}$ \\
\hline $\mathrm{m}_{R}$ limit & 12.5 & 15 \\
$\mathrm{~m}_{H}$ limit & 11 & 13 \\
$\mathrm{~m}_{H}$ correlated & 11.5 & 13.5 \\
$\begin{array}{l}\text { magnitude limit } \\
\text { Resolution } \\
\text { (milli-arcsec) }\end{array}$ & 0.8 & 1.2 \\
$\begin{array}{l}\text { Contrast } \\
\text { Imaging Field } \\
\text { of View } \\
\text { (milli-arcsec) }\end{array}$ & $\begin{array}{l}1000: 1 \\
10-100\end{array}$ & $1000: 1$ \\
& & 15
\end{tabular}

per telescope. This can be converted to tilt and piston, but fails whenever piston reaches plus or minus half a wave. For targets that differ significantly from a point source, the technique also requires a prior model of the target visibility function. We have not yet added this functionality to our simulator, but it it is well tested in the literature (e.g. N'Diaye paper in the adaptive optics conference of this SPIE), and as can be seen below - tilt and piston corrections can be computed and applied robustly even in the nonlinear regime, bringing higher order aberrations to the required linear regime for this additional technique. 


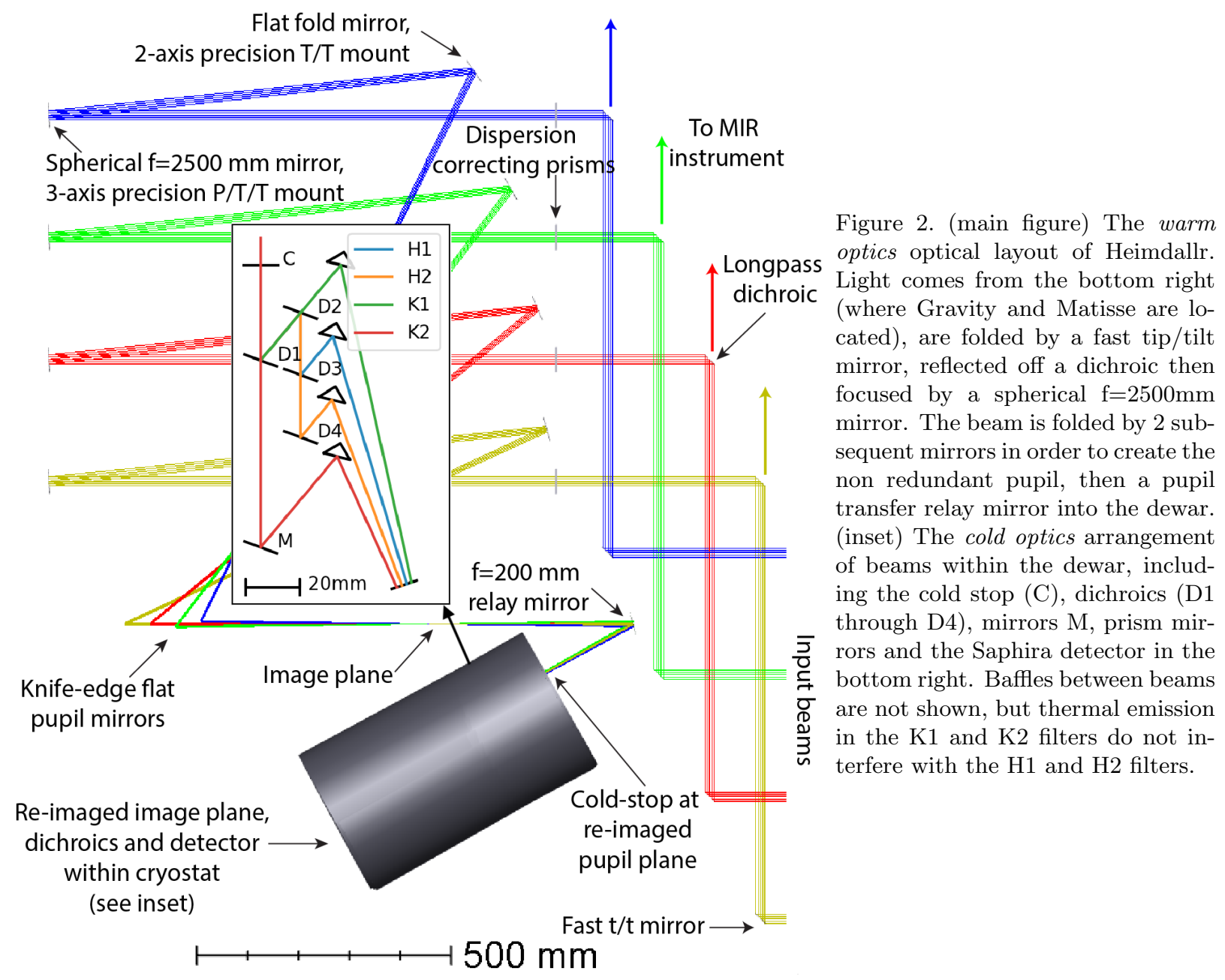

\subsection{Discrete Fourier Fringe Measurements}

By fitting in the Fourier plane and de-modulating the fringes from each baseline, we can determine tilt and piston on each sub-aperture separately, e.g. as was done for the Keck segment-tilting experiment, ${ }^{19}$ and can acquire fringes at many radians of phase offset. In addition, pupil alignment drifts can be computed and corrected for by measuring the frequency of the fringes on each baseline accurately. Finally, once these main terms are locked, higher-order aberrations can be found and corrected for using the linear assymetric wavefront sensor algorithm. Note that this algorithm requires strict redundancy between baselines in the pupil, which uses more pixels than a readout-noise optimal linear assymetric pupil wavelength sensor. We describe the algorithm for piston, tilt and pupil position separately.

\subsubsection{Piston Measurements}

The key observable in measurement if piston is the complex visibility, $V_{i j}$ between telescopes $i$ and $j$, combined with its uncertainty $\Delta V_{i j}$. Within a Bayesian framework, the problem of piston estimation then becomes a problem of estimating telescope pistons given both current and past baseline visibilities:

$$
P\left(p_{1}\left(t_{n+1}\right), p_{2}\left(t_{n+1}\right), \ldots \mid\left\{V_{12}\left(t_{n}\right), V_{13}\left(t_{n}\right), \ldots\right\},\left\{V_{12}\left(t_{n-1}\right), V_{13}\left(t_{n-1}\right), \ldots\right\}, \ldots\right) .
$$

We approximate the complex visibility as producing a phase measurement $x_{i j}=\operatorname{Arg}\left(V_{i j}\right)$ with an uncertainty $\Delta x_{i j}=\Delta V_{i j} /\left|V_{i j}\right|$ and a non-Gaussian probability density function, approximated in our initial simulations as: 

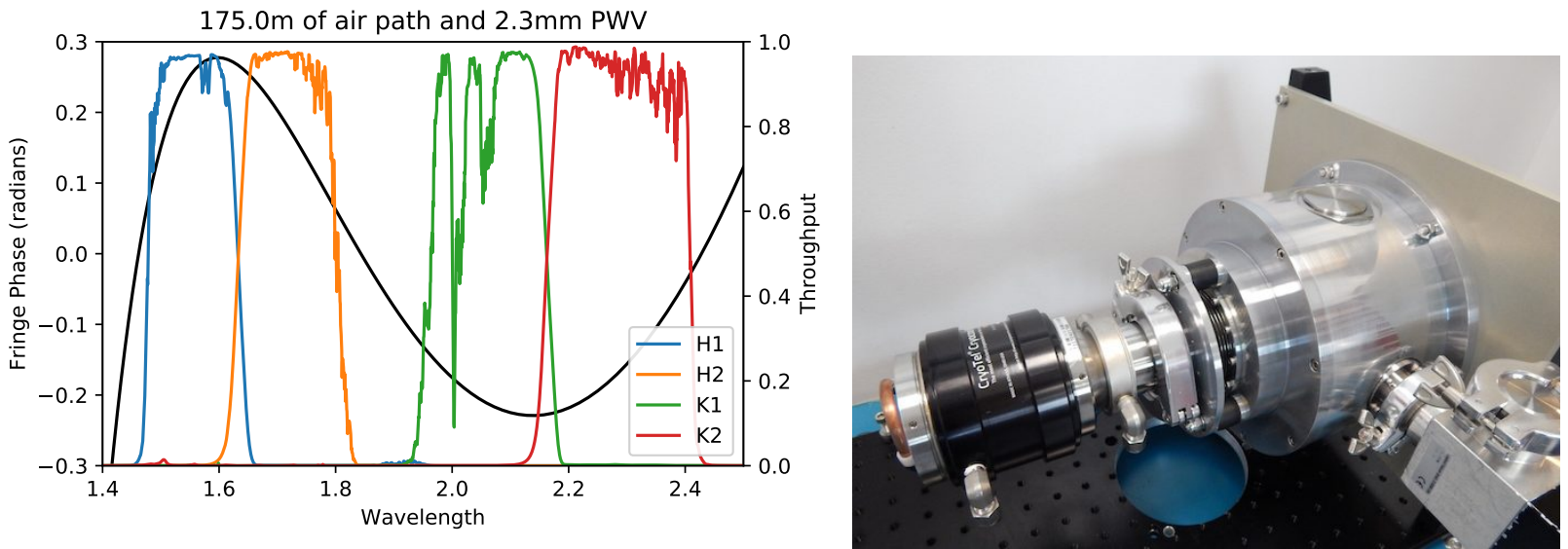

Figure 3. (left) The four bandpasses of Heimdallr, based on the coating design by Asahi Spectra, combined with the atmospheric transmission at median Paranal precipitable water vapour, and the fringe-phase after dispersion compensation by ZnSe prisms at the maximum pathlength (black). (right) The Heimdallr cryostat will be an only slightly modified version of the cryostat already in succesful operation with a Saphira detector at ANU. The cryostat design will be lengthened by $100 \mathrm{~mm}$ to fit the cryogenic optics plate.
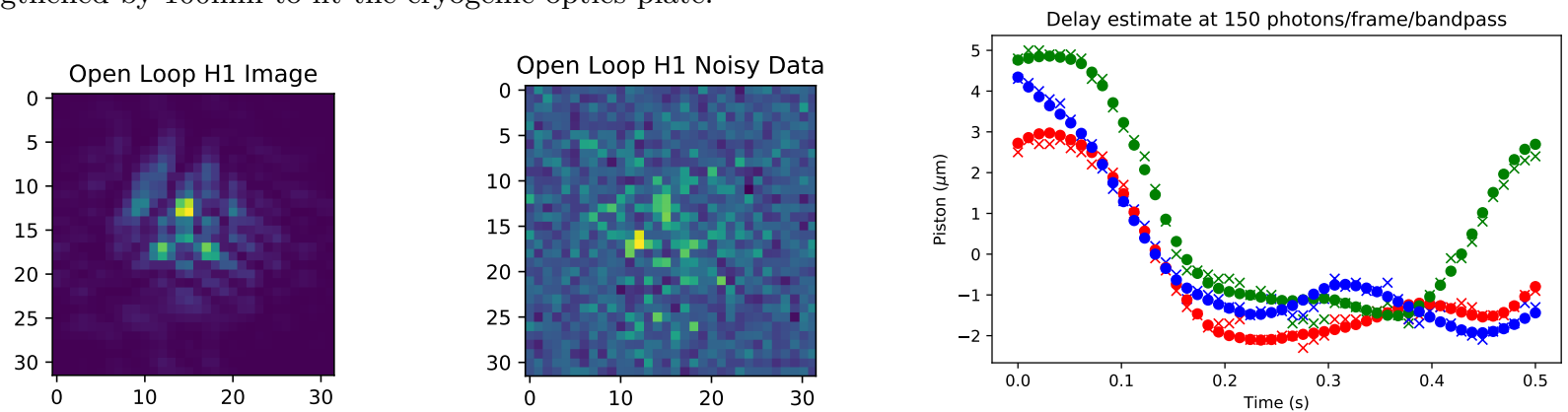

Figure 4. (left) A typical simulator output for open-loop fringe acquisition, without noise, but showing effects of imperfect adaptive optics. (center) The same image once noise is added (150 target photons/frame. (right) Open loop optical delay (circles) compared with delay estimates (crosses) at this low flux level. It is the performance of our complete simulator that gives us confidence that the magnitude limits in Table 1 are realistic (and somewhat conservative).

$$
\begin{aligned}
f_{\phi}\left(\phi_{i j}\right) & \propto \exp \left(\frac{\left.d\left(\phi_{i j}, x_{i j}\right)\right)^{2}}{2 \Delta x_{i j}^{2}}\right)+0.05 \exp \left(\frac{1}{2 \Delta x_{i j}^{2}}\right), \text { where } \\
d\left(\phi_{i j}, x_{i j}\right) & =\left(\left(\phi_{i j}-x_{i j}+\pi\right) \bmod 2 \pi\right)-\pi .
\end{aligned}
$$

With 6 differential piston measurements from 6 baselines and 4 simultaneous wavelength channels $\lambda_{k}$, we then explicitly calculate the joint probability density function $f_{p}$ of the telescope pistons $\left(p_{0}, p_{1}, p_{2}, p_{3}\right)$ with $p_{0}$ explicitly set to 0 :

$$
\begin{aligned}
f_{p}\left(p_{1}, p_{1}, p_{3}\right) & =\Pi_{i, j, k} f\left(\phi_{i j}(k)\right), \text { with } \\
\phi_{i j}(k) & =2 \pi\left(p_{j}-p_{i}\right) / \lambda_{k}
\end{aligned}
$$

The maximum in the likelihood function then gives the positions of the delays. This approach also enables the results of many subsequent exposures to be used optimally, and is the approach used in the simulator shown in Figure 4. In a simple model with white piston noise, the likelihood function is convolved by a multi-dimensional 
Gaussian distribution of standard deviation $\lambda_{0}\left(\Delta t / \tau_{0}\right)^{5 / 6}$, where $\tau_{0}$ is an estimate of the atmospheric coherence time at wavelength $\lambda_{0}$. It rapidly becomes computationally intensive for large numbers of telescopes, but appears to be well within the capabilities of a single GPU with up to 9 telescopes.

\subsubsection{Tilt Measurements}

Tilts can be derived in a similar way to the Keck segment tilting experiment. ${ }^{19}$ In short - the power from each baseline is windowed in the two-dimensional Fourier transform of the data, then inverse transformed. This produces fringes within an envelope with a centroid equal to the mean of the telescope tip/tilt centroid for the two telescopes that make up that baseline. The centroid of the square modulus of each of these fringe envelopes is found, e.g. $B_{1 x}$ for the $x$ direction centroid for baseline 1, joining telescopes 1 and 2 . The total intensity centroids $I_{x}$ and $I_{y}$ are also found. The telescope tilts are then found by a maximum likelihood combination of these centroids, for example for telescope 1 with uniform visibility on all baselines, we arrive at:

$$
\begin{aligned}
T_{1 x} & =\frac{1}{11}[8,6,6,6,-5,-5,-5] \\
& \cdot\left[I_{x}, B_{1 x}, B_{2 x}, B_{3 x}, B_{4 x}, B_{5 x}, B_{6 x}\right]^{\top}
\end{aligned}
$$

In the case of unit visibility, this centroid estimate has a shot-noise limited uncertainty only $\sim 2.5$ times higher than using all the flux from each telescope in ideal tip/tilt sensors (i.e. SNR is degraded by approximately the square root of the number of telescopes). We have verified that this approach works to lock a simulated servo loop with Strehl ratios approximately $50 \%$ and 100 photons per bandpass per frame.

\subsubsection{Pupil Alignment}

Metrology of the relative pupil positions within a redundant pupil fizeau beam combiner is simply a matter of measuring the positions of peak Fourier power (the "splodge" locations) for each baseline. Using a slow servo loop, these relative pupil positions can be fed back to a pupil alignment servo loop as needed (internally or externally).

\section{DISCUSSION}

In this paper, we introduced a simple non-redundant pupil all-in-one beam combiner detecting fringes in several filters as an efficient fringe tracker. Such a fringe tracker can also be used for alignment (image-plane and pupil-plane), low-order adaptive optics non common path correction and in some circumstances, fast tip/tilt.

A key weakness in the use of information from a fringe tracker in feeding back to adaptive optics systems at individual telescopes is that signal-to-noise is proportional to the mean fringe visibility of all baselines that include each telescope. For a well-resolved target, the individual telescope adaptive optics systems therefore become essential as sensors for all modes, including tip/tilt. However, low sensitivity in detecting well-resolved fringes is not necessarily a clear reason for changing a wavelength split between fringe tracking and adaptive optics wavefront sensing in favour of the adaptive optics system. For may applications, including spectrally resolved precision measurements of spatially resolve stellar atmospheres, the key requirement will still be that fringe phase measurement error is of order 1 radian in a coherence time for each telescope.

Further work on this concept will include a more complete simulation that includes a full adaptive optics system including sensor noise, and slow feedback from linear asymmetric wavefront sensing to correct non common path aberrations. In addition, we aim to a direct comparison to fringe tracking with single-mode fibers, to evaluate if spatial filtering by fibers has any practical advantage over spatial filtering by windowing the data in our image-plane fringe tracking algorithm, and to determine how appropriate windowing can aid fringe tracking for a longer-wavelength beam combiner which is itself spatially filtered.

\section{ACKNOWLEDGMENTS}

MI acknowledges support from the Australian Research Council's future fellowship scheme (FT130100235). 


\section{REFERENCES}

[1] Eisenhauer, F., Perrin, G., Brandner, W., Straubmeier, C., Richichi, A., Gillessen, S., Berger, J. P., Hippler, S., Eckart, A., Schöller, M., Rabien, S., Cassaing, F., Lenzen, R., Thiel, M., Clénet, Y., Ramos, J. R., Kellner, S., Fédou, P., Baumeister, H., Hofmann, R., Gendron, E., Boehm, A., Bartko, H., Haubois, X., Klein, R., Dodds-Eden, K., Houairi, K., Hormuth, F., Gräter, A., Jocou, L., Naranjo, V., Genzel, R., Kervella, P., Henning, T., Hamaus, N., Lacour, S., Neumann, U., Haug, M., Malbet, F., Laun, W., Kolmeder, J., Paumard, T., Rohloff, R. R., Pfuhl, O., Perraut, K., Ziegleder, J., Rouan, D., and Rousset, G., "GRAVITY: getting to the event horizon of Sgr A*," in [Optical and Infrared Interferometry], 7013, 70132A (July 2008).

[2] Quirrenbach, A., Mozurkewich, D., Buscher, D. F., Hummel, C. A., and Armstrong, J. T., "Phase-referenced visibility averaging in optical long-baseline interferometry," A\&A 286, 1019-1027 (June 1994).

[3] Perraut, K., Dougados, C., Lima, G. H. R. A., Benisty, M., Mourard, D., Ligi, R., Nardetto, N., Tallon-Bosc, I., ten Brummelaar, T., and Farrington, C., "A disk wind in AB Aurigae traced with H $\alpha$ interferometry," A\&A 596, A17 (Nov. 2016).

[4] Muterspaugh, M. W., Lane, B. F., Kulkarni, S. R., Konacki, M., Burke, B. F., Colavita, M. M., Shao, M., Wiktorowicz, S. J., and O'Connell, J., "The Phases Differential Astrometry Data Archive. I. Measurements and Description," AJ 140, 1579-1622 (Dec. 2010).

[5] Kok, Y., Ireland, M. J., Tuthill, P. G., Robertson, J. G., Warrington, B. A., Rizzuto, A. C., and Tango, W. J., "Phase-Referenced Interferometry and Narrow-Angle Astrometry with SUSI," Journal of Astronomical Instrumentation 2, 1340011 (Jan. 2013).

[6] Defrère, D., Absil, O., Berger, J. P., Boulet, T., Danchi, W. C., Ertel, S., Gallenne, A., Hénault, F., Hinz, P., Huby, E., Ireland, M., Kraus, S., Labadie, L., Le Bouquin, J. B., Martin, G., Matter, A., Mérand, A., Mennesson, B., Minardi, S., Monnier, J., Norris, B., Orban de Xivry, G., Pedretti, E., Pott, J. U., Reggiani, M., Serabyn, E., Surdej, J., Tristram, K. R. W., and Woillez, J., "The path towards high-contrast imaging with the VLTI: the Hi-5 project," ArXiv e-prints, arXiv:1801.04148 (Jan. 2018).

[7] Monnier, J. e. a., "The planet formation imager," Experimental Astronomy accepted, - (2018).

[8] Martinache, F. and Ireland, M. J., "Kernel-nulling for a robust direct interferometric detection of extrasolar planets," ArXiv e-prints, arXiv:1802.06252 (Feb. 2018).

[9] Guyon, O., "Limits of Adaptive Optics for High-Contrast Imaging," ApJ 629, 592-614 (Aug. 2005).

[10] Widmann, F., Pott, J.-U., and Velasco, S., "P-REx: The Piston Reconstruction Experiment for infrared interferometry," MNRAS 475, 1224-1237 (Mar. 2018).

[11] Sarazin, M. and Tokovinin, A., "The Statistics of Isoplanatic Angle and Adaptive Optics Time Constant derived from DIMM Data," in [European Southern Observatory Conference and Workshop Proceedings], Vernet, E., Ragazzoni, R., Esposito, S., and Hubin, N., eds., European Southern Observatory Conference and Workshop Proceedings 58, 321 (2002).

[12] Finger, G., Baker, I., Alvarez, D., Dupuy, C., Ives, D., Meyer, M., Mehrgan, L., Stegmeier, J., and Weller, H. J., "Sub-electron read noise and millisecond full-frame readout with the near infrared eAPD array SAPHIRA," in [Adaptive Optics Systems V], Proc. SPIE 9909, 990912 (July 2016).

[13] Jovanovic, N., Schwab, C., Guyon, O., Lozi, J., Cvetojevic, N., Martinache, F., Leon-Saval, S., Norris, B., Gross, S., Doughty, D., Currie, T., and Takato, N., "Efficient injection from large telescopes into single-mode fibres: Enabling the era of ultra-precision astronomy," A\&A 604, A122 (Aug. 2017).

[14] Ireland, M. J., Monnier, J. D., Kraus, S., Isella, A., Minardi, S., Petrov, R., ten Brummelaar, T., Young, J., Vasisht, G., Mozurkewich, D., Rinehart, S., Michael, E. A., van Belle, G., and Woillez, J., "Status of the Planet Formation Imager (PFI) concept," in [Optical and Infrared Interferometry and Imaging V], 9907, 99071L (Aug. 2016).

[15] Petrov, R. G., Boskri, A., Elhalkouj, T., Monnier, J., Ireland, M., and Kraus, S., "Co-phasing the planet formation imager," in [Optical and Infrared Interferometry and Imaging V], 9907, 99073W (Aug. 2016).

[16] Ireland, M. J., Mérand, A., ten Brummelaar, T. A., Tuthill, P. G., Schaefer, G. H., Turner, N. H., Sturmann, J., Sturmann, L., and McAlister, H. A., "Sensitive visible interferometry with PAVO," in [Optical and Infrared Interferometry], Proc. SPIE 7013, 701324 (July 2008). 
[17] Martinache, F., Jovanovic, N., and Guyon, O., "Closed-loop focal plane wavefront control with the SCExAO instrument," A\&A 593, A33 (Sept. 2016).

[18] Tango, W. J., "Dispersion in stellar interferometry," Appl. Opt. 29, 516-521 (Feb. 1990).

[19] Monnier, J. D., Tuthill, P. G., Ireland, M., Cohen, R., Tannirkulam, A., and Perrin, M. D., "Mid-Infrared Size Survey of Young Stellar Objects: Description of Keck Segment-Tilting Experiment and Basic Results," ApJ 700, 491-505 (July 2009). 